\title{
Different plantar flexors neuromuscular and mechanical characteristics depending on the preferred running form
}

\author{
Sidney Grosprêtre ${ }^{\mathrm{a}, *}$, Philippe Gimenez ${ }^{\mathrm{a}}$, Adrien Thouvenot ${ }^{\mathrm{b}, \mathrm{c}}$, Aurélien Patoz ${ }^{\mathrm{d}}$, \\ Thibault Lussiana ${ }^{\mathrm{b}, \mathrm{c}}$, Laurent Mourot $^{\mathrm{c}, \mathrm{e}}$ \\ ${ }^{\text {a }}$ EA4660-C3S Laboratory - Culture, Sports, Health and Society, Univ. Bourgogne Franche-Comté, Besançon, France \\ ${ }^{\mathrm{b}}$ Research and Development Department, Volodalen, Chavéria, France \\ ${ }^{\mathrm{c}}$ EA3920-Prognostic Markers and Regulatory Factors of Heart and Vascular Diseases, and Exercise Performance, Health, Innovation Platform, Univ. Bourgogne Franche- \\ Comté, Besançon, France \\ ${ }^{\mathrm{d}}$ Research and Development Department, Volodalen Swiss Sport Lab, Aigle, Switzerland \\ ${ }^{\mathrm{e}}$ National Research Tomsk Polytechnic University, Tomsk, Russia
}

\section{A R T I C L E I N F O}

\section{Keywords:}

Triceps surae

Electromyography

Rate of force development

Twitch

H-reflex

Running

\begin{abstract}
A B S T R A C T
Two main types of endurance runners have been identified: aerial runners (AER), who have a larger flight time, and terrestrial runners (TER), who have a longer ground contact time. The purpose of this study was to assess the neuromuscular characteristics of plantar flexors between AER and TER runners. Twenty-four well-trained runners participated in the experiment. They were classified either in a TER or AER group according to the Volodalen ${ }^{\circledR}$ scale. Plantar flexors' maximal rate of force development (RFD) and maximal voluntary contraction force (MVC) were assessed. Percutaneous electrical stimulation was delivered to the posterior tibial nerve to evoke maximal M-waves and H-reflexes of the triceps surae muscles. These responses, as well as voluntary activation, muscle potentiation, and V-waves, were recorded by superimposing stimulations to MVCs. RFD was significantly higher in AER than in TER, while MVC remained unchanged. This was accompanied by higher myoelectrical activity recorded in the soleus muscle. While M-waves and other parameters remained unchanged, maximal $\mathrm{H}$ reflex was significantly higher in AER than in TER, still in soleus only. The present study raised the possibility of different plantar flexors' neuromuscular characteristics according to running profile. These differences seemed to be focused on the soleus rather than on the gastrocnemii.
\end{abstract}

\section{Introduction}

Sport practice has several long-term effects on the musculoskeletal system and neural network. When comparing athletes from untrained individuals, athletes showed greater force production capacity, as evidenced by higher rates of force development (RFD) (Tillin et al., 2010) and higher central activation (Ahtiainen and Häkkinen, 2009). Regarding some specific neural parameters, such as spinal excitability, the direction of the change depends upon the type of physical activity. Indeed, while power athletes usually exhibit lower values than untrained people, endurance athletes show greater spinal excitabilities
(Maffiuletti et al., 2001).

In most of the previous literature, endurance athletes from various sports (e.g., cycling, running, triathlon, cross-country skiing) have been pooled together and compared to power athletes (mixing sprinters, jumpers, or throwers). However, it could be hypothesized that different neuromuscular profiles also exist when considering a narrower portion of the continuum between endurance- and power-type athletes, i.e. targeting inter-individual differences within the same activity. Specifically, endurance running appears to be an activity for which various running forms may exist among its practitioners, e.g., ranging from "Groucho" to "Pose" running styles (Arendse et al., 2004; McMahon

Abbreviations: AER, Group of aerial runners; TER, Group of terrestrial runners; MVC, Maximal Voluntary Contraction; V®score, Global Subjective Score; VAL,

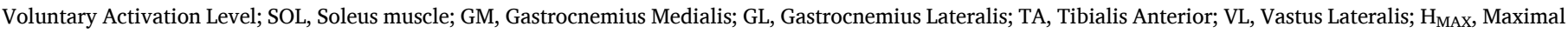

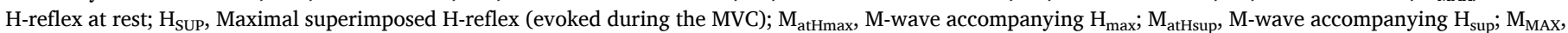

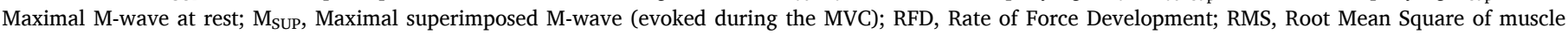
electromyographic activity (EMG); EME, Electro-mechanical Efficiency; PT, Peak of the single twitch.

* Corresponding author at: EA4660-CS3 “Culture, Sport, Health and Society”, UPFR Sport, 31, chemin de l'Epitaphe, 25000 Besançon, France.

E-mail address: sidney.grospretre@univ-fcomte.fr (S. Grosprêtre). 
et al., 1987).

Objective (Hoerzer et al., 2015; Lussiana et al., 2019; Phinyomark et al., 2015) and subjective (Lussiana et al., 2017b) kinematic and spatio-temporal differences between endurance runners have then been revealed. The terms Terrestrial (TER) and Aerial (AER) runners have been proposed to characterize these different running forms (Gindre et al., 2016). These TER and AER runners have been shown to have the same running economy (Lussiana et al., 2019, 2017a). To minimize the cost of movement, TER favour a long contact time associated with a rearfoot strike pattern, whereas AER favour a long flight time together with a forefoot strike pattern (Lussiana et al., 2019, 2017a). These observations were accompanied by an earlier activation of the gastrocnemius lateralis (GL) in preparation for landing in AER compared to TER (Lussiana et al., 2017a). Hence, the plantar flexor muscles group seems to be a key muscle group affected by the type of running pattern. Moreover, these muscles are one of the main investigated muscle groups in neuromuscular studies (Tucker et al., 2005). Early works using a similar approach at the ankle joint already identified links between kinematic variables and structural / neural properties of the plantar flexors of short and long-distance runners (Bach et al., 1983). The authors concluded that both neural and mechanical factors were key factors in determining the running pattern. Therefore, the purpose of the present study was to compare the neuromuscular and mechanical properties of the plantar flexors between AER and TER during a nonspecific task. As AER have shorter ground contact time and forefoot strike pattern, we hypothesized that they would exhibit greater spinal excitability compared to TER that have longer ground contact time, possibly reflecting higher mechanical load on the plantar flexor muscles.

\section{Material and methods}

\subsection{Participants}

Twenty-four well-trained healthy participants (7 women and 17 men) gave written informed consent to participate in the present experiment (Table 1). None of them reported neurological, physical disorders, or previous lower-limb injury in the previous six months. All experimental procedures were performed in accordance with the latest version of the Declaration of Helsinki and approved by the Regional Ethics Committee (CPP Est I 2016-A00511-50).

\subsection{Experimental design}

The experiment was carried out in a single session ( $1 \mathrm{~h} 45 \mathrm{~min}$ ), which included two parts performed randomly: an evaluation of the participant's running form (15 $\mathrm{min}$ ) and a neuromuscular and mechanical evaluation ( $1 \mathrm{~h} 30 \mathrm{~min}$ ). Both parts were performed in separate rooms with different operators, in a double-blind set-up : participants and operators were not informed of the results of the other experimental part (neuromuscular and runner's classification).

Table 1

Participant characteristics for Terrestrial (TER) and Aerial (AER) groups. Note: Data are means \pm S.D. M: Male, F: Female, and NA: statistical test not applicable.

\begin{tabular}{llll}
\hline & TER & AER & P value \\
\hline Age (y) & $29.6 \pm 10.5$ & $25.4 \pm 8.0$ & 0.286 \\
Height (m) & $1.71 \pm 0.07$ & $1.76 \pm 0.06$ & 0.213 \\
Mass (kg) & $64.2 \pm 9.2$ & $62.1 \pm 7.5$ & 0.551 \\
Training experience (y) & $5.9 \pm 3.8$ & $6.8 \pm 5.9$ & 0.658 \\
Running time (h/week) & $4.9 \pm 1.9$ & $7.1 \pm 3.6$ & 0.072 \\
Running distance (km/week) & $42.5 \pm 12.1$ & $51.7 \pm 16.5$ & 0.136 \\
Maximum aerobic speed (km/h) & $17.2 \pm 1.3$ & $18.4 \pm 1.8$ & 0.084 \\
V®score & $11.7 \pm 1.9$ & $20.2 \pm 2.2$ & NA \\
Sex & $\mathrm{M}=6 ; \mathrm{F}=6$ & $\mathrm{M}=11 ; \mathrm{F}=1$ & $\mathrm{NA}$ \\
\hline
\end{tabular}

\subsection{Runner's classification}

Participants performed a 5-min running warm-up at a self-selected speed, followed by a 10-min running trial at $12 \mathrm{~km} \cdot \mathrm{h}^{-1}$ on a treadmill (Training Treadmill S1830, HEF Techmachine, Andrézieux-Bouthéon, France). Two running coaches with several years of experience using the Volodalen ${ }^{\circledR}$ scale focused on the overall movement form of participants as they ran. The coaches paid attention to five key elements: vertical oscillation of the head, antero-posterior motion of the elbows, pelvis position at ground contact, antero-posterior foot position at ground contact, and foot strike pattern (Gindre et al., 2016; Lussiana et al., 2017a). The intra- and inter-rater reliability of this method has been shown recently (Patoz et al., 2019). Each element was scored from one to five, leading to a global subjective score $(\mathrm{V} \circledast s c o r e)$ that ultimately allows the classification of runners into TER $(\mathrm{V} \otimes$ score $\leq 15)$ or AER $\left(\mathrm{V}{ }^{\circledR}\right.$ score $\left.>15\right)$ group.

\subsection{Force recordings}

Participants seated in a comfortable experimental chair with hip, knee and ankle joints at $90^{\circ}$. The ankle was firmly strapped to a pedal equipped with a constraint gauge (PCE instruments, Soultz-Sous-Forets France). They were instructed to keep their hands free and to maintain the trunk against chair's back. The recording of one antagonist (i.e., tibialis anterior, TA) and one knee extensor (i.e., vastus lateralis, VL) allowed to monitor the contribution of other muscle groups to the developed force.

Participants were first asked to warm-up by performing eight to ten sub-maximal isometric plantar flexions at a progressive force level. Then, they performed eight isometric plantar flexion maximal voluntary contractions (MVC) of about $4 \mathrm{~s}$, separated by one-minute rest. During the plateau of each MVC, one nerve stimulation was triggered. The recording of RFD was assessed separately from MVC, as previously recommended (Maffiuletti et al., 2016). Participants were asked to "push hard and fast" up to their maximal force, without maintaining it. A total of eight to ten trials was performed, with $30 \mathrm{~s}$ rest in-between. Trials with countermovement (dorsi-flexion preceding the start of the plantar flexion) were excluded.

The mechanical signals were digitized on-line (sampling frequency: $2 \mathrm{kHz}$ ) and simultaneously recorded with electromyography of the targeted muscles. Signals were stored for analysis in Labchart software (LabChart 8, ADInstruments, Sydney, Australia).

\subsection{Electromyographic activity}

Electromyographic activity (EMG) was recorded from four muscles of the right leg (soleus, SOL; gastrocnemius medialis, GM; gastrocnemius lateralis, GL; TA; VL). The skin was first shaved and dry-cleaned with alcohol to keep low impedance $(<5 \mathrm{k} \Omega$ ). EMG signals were recorded with Trigno sensors (Delsys, Natick, Massachusetts, USA), firmly strapped to the leg with a skin rubber. Sensors were placed according to SENIAM recommendations (Hermens et al., 2000). EMG signals were amplified with a bandwidth frequency ranging from $0.3 \mathrm{~Hz}$ to $2 \mathrm{kHz}$ (gain: 1000) and digitized on-line (sampling frequency: $2 \mathrm{kHz}$ ) with Labchart software (LabChart 8, ADInstruments, Sydney, Australia).

\subsection{Electrical nerve stimulation}

The posterior tibial nerve was stimulated through single rectangular pulses (1-ms duration) delivered by a constant-current stimulator (Digitimer DS7A, Hertfordshire, UK). Stimulations were elicited with a self-adhesive cathode (8-mm diameter, Ag-AgCL) placed in the popliteal fossa and an anode $(5 \times 10 \mathrm{~cm}$, Medicompex SA, Ecublens, Switzerland) placed over the patella. Once the optimal spot was determined, the stimulation electrode was firmly fixed to this site with straps. The intensity of the stimulation was then progressively increased from SOL, 
GM and GL H-reflex threshold with $2 \mathrm{~mA}$ increment to maximal H-reflex $\left(\mathrm{H}_{\mathrm{MAX}}\right)$ and then with $5 \mathrm{~mA}$ increment until M-wave of the three muscles no longer increased. This last stimulation-intensity was then increased by $20 \%$ to record maximal $\mathrm{M}$-wave $\left(\mathrm{M}_{\mathrm{MAX}}\right)$. Four stimulations were performed at each intensity.

\subsection{Data analyses}

All data were stored, synchronized and analyzed in LABCHART 8 software (LabChart 8, ADInstruments, Sydney, Australia).

\subsection{Mechanical data}

The RFD was analysed as the derivate of the mechanical signal (in $\mathrm{N} \cdot \mathrm{sec}^{-1}$ ) during the contraction ramp, i.e., from the baseline to the peak of force produced. The mean of the best three trials was analysed.

Maximal isometric force was taken as the peak of the mechanical signal obtained during the plateau of MVC prior to the stimulus artefact. Voluntary activation level (VAL) was determined using the twitch interpolation technique by using the following formula

$\mathrm{VAL}=100\left[1-\left(\frac{\mathrm{PT}_{\mathrm{S}}}{\mathrm{PT}_{\mathrm{P}}}\right)\right]$,

where $\mathrm{PT}_{\mathrm{S}}$ is the superimposed force amplitude induced by the stimulation at $\mathrm{M}_{\mathrm{MAX}}$ intensity during the $\mathrm{MVC}$. $\mathrm{PT}_{\mathrm{P}}$ is the potentiated twitch force amplitude taken as the mechanical peak evoked at $\mathrm{M}_{\mathrm{MAX}}$ following MVC.

Triceps surae potentiation was expressed as the change (\%) between the amplitude of the resting twitch $\left(\mathrm{PT}_{\mathrm{R}}\right)$ and $\mathrm{PT}_{\mathrm{P}}$

Potentiation $=100\left[\frac{\left(\mathrm{PT}_{\mathrm{P}}-\mathrm{PT}_{\mathrm{R}}\right)}{\mathrm{PT}_{\mathrm{R}}}\right]$

\subsection{Electrophysiological data}

The root mean square (RMS) value of SOL, GM and GL muscles EMG signals were determined with an integration time of $500 \mathrm{~ms}$ over the plateau during plantar flexion MVCs, prior to the stimulus artifacts. SOL and GM RMS were normalized by the corresponding $M_{S U P}$. During these RFDs, the contribution of each triceps surae muscle was calculated as the

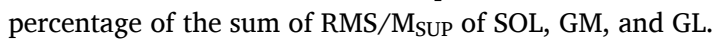

Peak-to-peak amplitudes of electromyographic responses at rest $\left(\mathrm{H}_{\text {MAX }}, \mathrm{M}_{\text {MAX }}\right)$ and during MVC ( $\left.\mathrm{H}_{\text {SUP }}, \mathrm{M}_{\text {SUP }}, \mathrm{V}\right)$ were measured for quantitative analysis. It can be noticed that maximal H-reflex, reflecting spinal excitability, is generally associated with a small M-wave (noted $\mathrm{M}_{\mathrm{atHmax}}$ at rest and $\mathrm{M}_{\mathrm{atHsup}}$ during MVC), which was also measured. Contrary to rest, it can be noticed that $\mathrm{M}_{\mathrm{SUP}}$ is followed by a reflexive response, called V-wave, which was used as an index of the supra-spinal descending neural drive (Grospretre and Martin, 2014). For each muscle, all responses were normalized to maximal $\mathrm{M}$-wave evoked in the same condition. Thus, $\mathrm{H}_{\text {MAX }} / \mathrm{M}_{\mathrm{MAX}}, \mathrm{M}_{\mathrm{atH} \max } / \mathrm{M}_{\mathrm{MAX}}, \mathrm{H}_{\mathrm{SUP}} / \mathrm{M}_{\mathrm{SUP}}$, $\mathrm{M}_{\mathrm{atHsup}} / \mathrm{M}_{\mathrm{SUP}}, \mathrm{V} / \mathrm{M}_{\text {SUP, }}$, were considered as dependent variables and compared between TER and AER.

Finally, the total electro-mechanical efficiency (EME) was determined by the ratio of the peak twitch evoked at $\mathrm{M}_{\max }(\mathrm{PT})$ over the sum of SOL and GM M-waves. EME reflects the excitation-contraction coupling efficiency.

\subsection{Statistical analyses}

All data are presented as the mean \pm standard deviation (S.D.). The normality of the data and the homogeneity of variances were confirmed through the Shapiro-Wilk and Levene's tests, respectively. Participant characteristics and dependent variables (mechanical and electrophysiological data) between AER and TER were analyzed through two-tailed unpaired Student's t-tests. A separate analysis was performed for each muscle, except for the percentage of muscle contributions during the RFD, which were gathered for analysis, by means of a two-way ANOVA with factor group (AER vs. TER) and muscle (SOL vs. GM vs. GL). Main effects and interactions were followed-up by post hoc HSD Tukey's tests. Statistical analysis was performed using STATISTICA (8.0 version, Statsoft, Tulsa, Okhlaoma, USA). The level of significance was set at $\mathrm{P}<$ 0.05 .

\section{Results}

\subsection{Characteristics of Aerial and Terrestrial runners}

The participant characteristics for TER and AER are given in Table 1. All baseline characteristics were similar between both groups.

\subsection{Mechanical data}

There was no between-group difference observed in MVC $(\mathrm{P}=0.541$; Fig. 1A), mechanical twitches $(\mathrm{P}=0.454$; Fig. 1B) or activation levels ( $\mathrm{P}$ $=0.888$; Fig. 1C). However, muscle potentiation was significantly higher for TER than for AER ( $\mathrm{P}=0.030$; Fig. 1D). No difference in EMG RMS recorded during MVC was found in any of the tested muscles between TER and AER (data not shown for the sake of clarity).

RFD was higher in AER than in TER $(P=0.030$, Fig. 2A). This greater RFD in AER was accompanied by greater normalized EMG RMS observed in SOL muscle, while no differences were observed in gastrocnemii (Fig. 2B, C and D). When expressed as a percentage of the total activation (sum of all RMS/M $\mathrm{M}_{\mathrm{SUP}}$ ), significant group $\times$ muscle interaction has been found for the relative contributions of each muscle to the RFD $(P=0.007)$, AER showing a significantly greater SOL $(P=0.010)$ and a significantly lower GM $(\mathrm{P}=0.047)$ muscles contribution to the RFD than TER (Fig. 2E).

\subsection{Electrophysiological data}

Firstly, no inter-group differences were found in muscle compound action potentials, for rest response $\left(\mathrm{M}_{\mathrm{MAX}}\right)$ as for superimposed one $\left(\mathrm{M}_{\mathrm{SUP}}\right)$, in any of the tested muscles. In addition, the submaximal Mwaves that accompanied $\mathrm{H}$-reflexes $\left(\mathrm{M}_{\mathrm{atHmax}}\right.$ and $\left.\mathrm{M}_{\text {atHsup }}\right)$ did not differ between groups for all muscles.

Secondly, normalized maximal $\mathrm{H}$-reflex at rest $\left(\mathrm{H}_{\mathrm{MAX}} / \mathrm{M}_{\mathrm{MAX}}\right)$ was significantly higher in AER than TER for SOL muscle $(\mathrm{P}=0.040$; Fig. 3A), but not for GM ( $P=0.475$; Fig. $3 \mathrm{~B})$ and GL $(\mathrm{P}=0.804$, Fig. $3 \mathrm{C})$. No significant differences were observed in superimposed H-reflexes ( $\mathrm{H}_{\mathrm{SUP}} / \mathrm{M}_{\mathrm{SUP}}$ ) (SOL: $\mathrm{P}=0.346$; GM: $\mathrm{P}=0.170 ; \mathrm{GL}=0.711$, Fig. 3). Since $\mathrm{V} / \mathrm{M}_{\mathrm{SUP}}$ did not significantly differ between both groups for each tested muscle (SOL: $\mathrm{P}=0.573$; GM: $\mathrm{P}=0.509$; GL: $\mathrm{P}=0.533$, Fig. 3 ), no significant differences were found at the supraspinal level.

\section{Discussion}

The present study aimed to compare the neuromuscular and mechanical properties of the plantar flexors between two groups of endurance runners with different preferred running forms. AER exhibited higher RFD compared to TER despite a similar maximal peak force. This was accompanied by greater changes in SOL EMG activity and H-reflex for AER, whereas peripheral (M-waves) and supraspinal (Vwaves) electrophysiological indexes were not different among groups. On the other hand, TER exhibited greater muscle potentiation, partly validating our hypothesis regarding this group.

\subsection{Different muscle contributions}

Leg muscles' contributions during running were different between AER and TER, as already shown during the running stance (Lussiana 


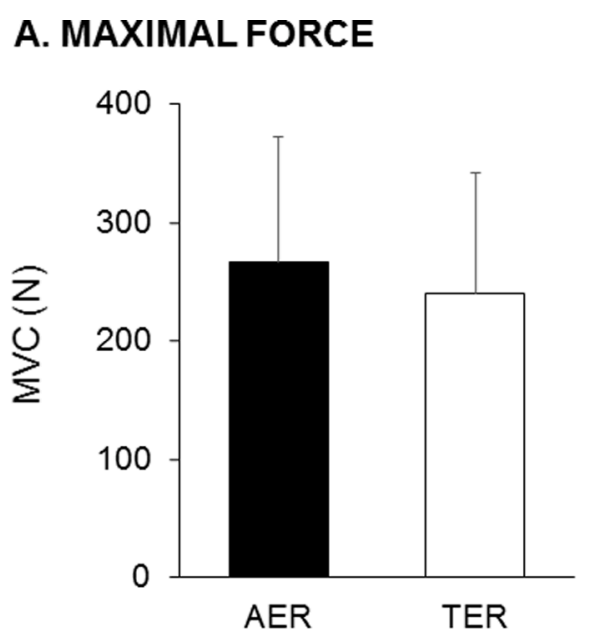

C. ACTIVATION LEVEL

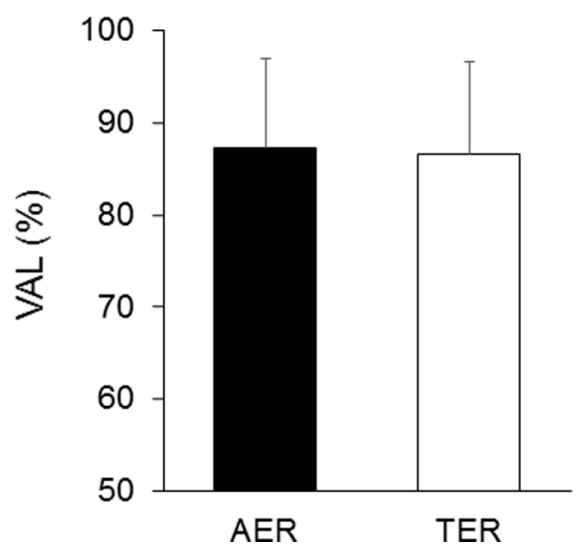

\section{B. MECHANICAL TWITCH}

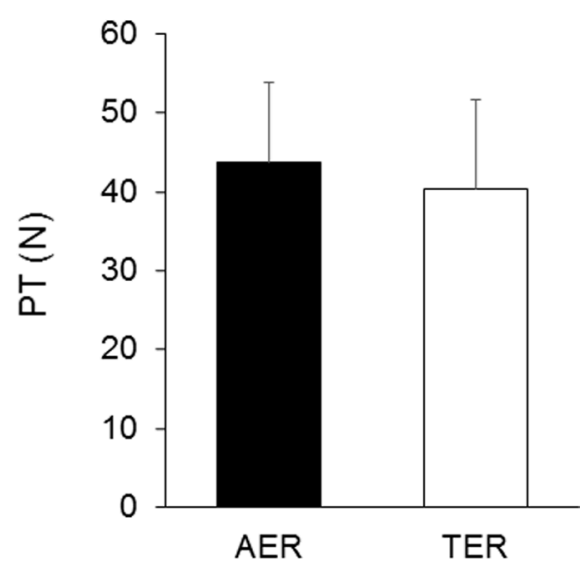

D. POTENTIATION

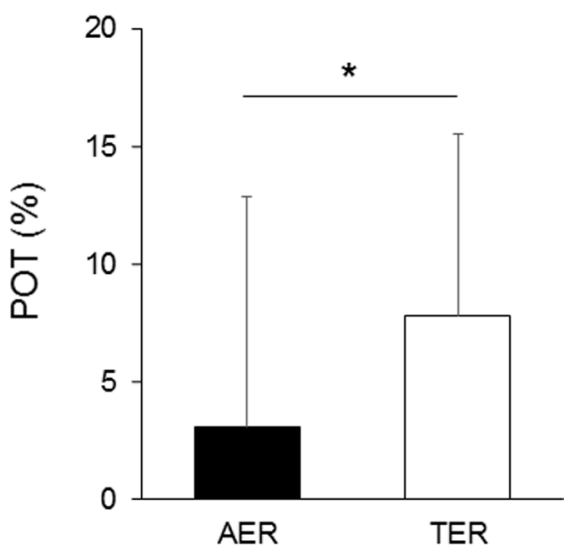

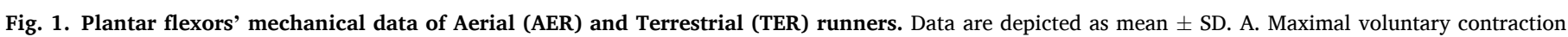

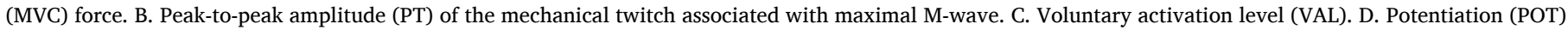
between the twitch evoked before the MVC and the twitch evoked after. *: significant inter-group difference at $\mathrm{P}<0.05$.

et al., 2017a). Concomitantly, it was shown that AER landed in a more plantar flexed position than TER. This suggests a greater contribution of the plantar flexor muscles in AER compared to TER, given its essential contribution at the ankle level. Accordingly, the results of the present study showed that AER exhibited a higher plantar flexor RFD compared to TER and the associated myoelectrical activities also argued in favor of a greater SOL contribution in such performance. AER is used to run with short contact time and large vertical oscillations. Therefore, for the same force production, AER could be able to support a greater RFD.

Given the fact that the tested performance was not running-specific, this result highlights a difference in general neural strategy. Our results showed that AER exhibited greater spinal excitability than TER in SOL muscle but not in gastrocnemii muscles. It has been shown that training of the plantar flexors can induce different adaptation in spinal excitabilities of SOL compared to GM (Duclay et al., 2008). These different adaptations can be attributed either to i) the different spinal network of SOL and gastrocnemii as a result of their different type of motor units, i. e. slow versus fast (Johnson et al., 1973) or ii) their difference in muscle spindles density, those which mediates the stretch reflex (Tucker et al., 2005). One interesting fact to notice is that one of the most effective modalities to induce such changes in spinal excitability and such discrepancy between muscles is eccentric training (Duclay et al., 2008). It was shown that SOL and gastrocnemii muscles exhibited different behavior in muscle fascicle stretch during plantar flexors eccentric actions but not always during concentric actions (Chino et al., 2008). These clues are in favor of greater eccentric load undertaken by AER.
Indeed, plantar flexors being in eccentric modality during the first part of the stance, a larger aerial phase and vertical oscillation during the gait cycle leads to a higher load to support at landing, especially because AER favored a forefoot strike pattern (Lussiana et al., 2019, 2017a). Finally, although no significant inter-group difference in RMS/M $\mathrm{M}_{\text {SUP }}$ was observed for the gastrocnemii, it should be noted that the relative contributions of GM displayed a difference, while GL did not (Fig. 3). This result raises the fact that $\mathrm{GM}$ is the only muscle to compensate for a greater activation of SOL since GL exhibited a similar contribution between AER and TER.

\subsection{Underlying mechanisms}

First of all, the lack of changes in MVC force, nor in VAL and V-wave amplitude, excludes a potential contribution of supraspinal levels to demonstrate the differences between the two groups. However, this does not preclude a more qualitative difference at a cortical level, such as different brain activations.

Regarding the RFD, a common opinion is to attribute a high performance to a high fast fibre proportion in the considered muscle group, being a marker of explosive muscle strength (Folland et al., 2014). Intersetingly, although slightly superior in power athletes, the plantar flexors' RFD did not exhibit any differences between endurance- and power-type athletes. (Kyröläinen and Komi, 1994). These authors suggested that the global muscle mass or typology, could not be the unique factor to affect maximal RFD. For instance, despite conflicting results 


\section{A. RATE OF FORCE DEVELOPMENT}

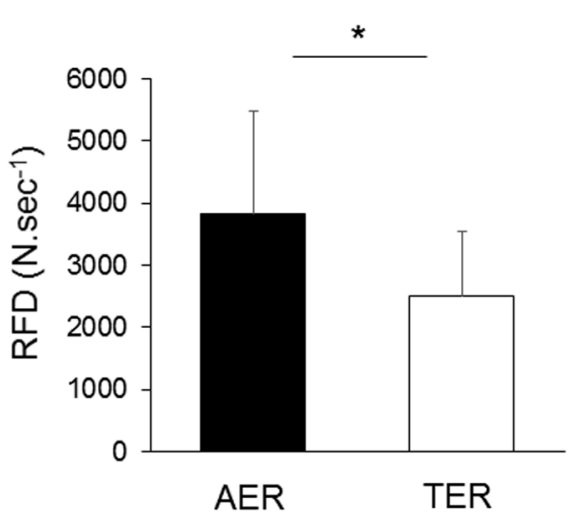

\section{C. $G M$}

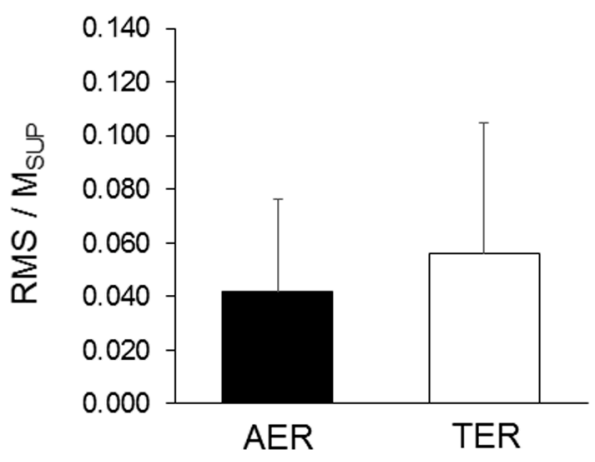

\section{E. CONTRIBUTION OFEACH MUSCLE}

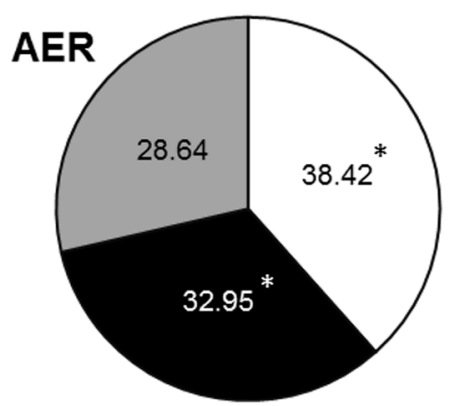

B. SOL

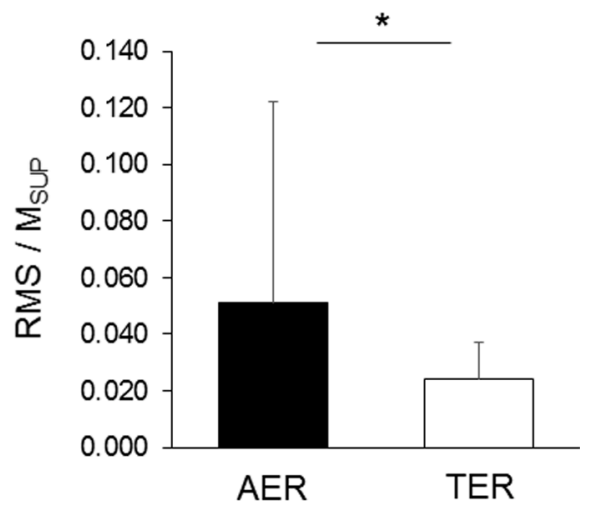

Fig. 2. Plantar flexors' rate of force development of Aerial (AER) and Terrestrial (TER) runners. Data are depicted as mean \pm SD. A. Rate of force development (RFD) in plantar flexion. B, C and D, shows the associated peak in electromyographic activities, expressed as the root mean square of the activity (RMS) normalized by the maximal active $\mathrm{M}$-wave $\left(\mathrm{M}_{\mathrm{SUP}}\right)$, for the soleus muscle (SOL), gastrocnemius medialis (GM) and gastrocnemius lateralis (GL), respectively. Panel $\mathrm{E}$ depicts the relative contribution of each muscle to the RFD, in \% of total muscle activity. *: significant intergroup difference at $\mathrm{P}<0.05$.

D. GL
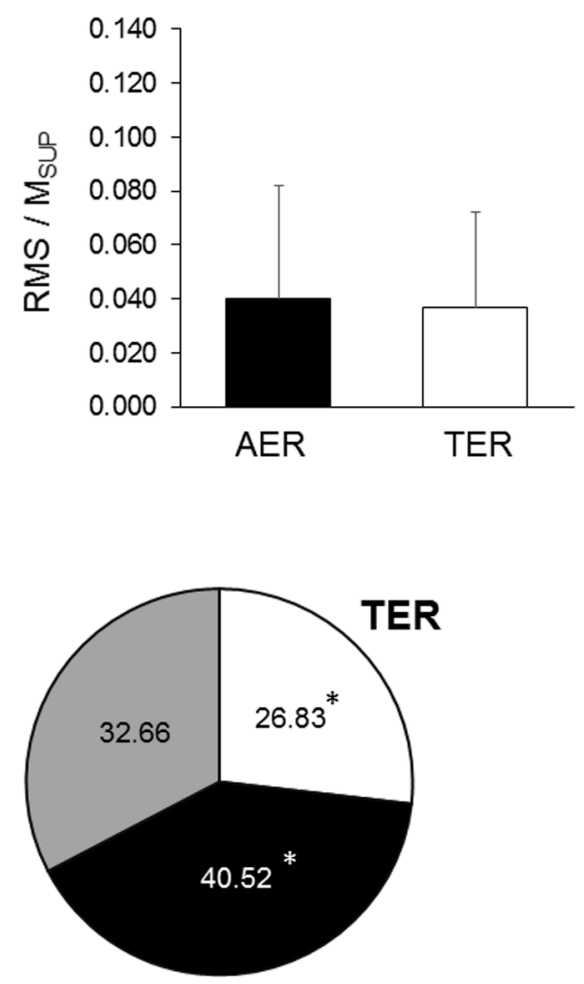

$\square S O L=G M \square G L$

(Buckthorpe and Roi, 2018; Buckthorpe and Roi, 2018; Buckthorpe and Roi, 2018; Buckthorpe and Roi, 2018; Buckthorpe and Roi, 2018; Buckthorpe and Roi, 2018; Buckthorpe and Roi, 2018) a close link between plantar flexors RFD and musculo-tendinous stiffness has been observed (Driss et al., 2012). Hence, our previous observations of greater leg stiffness in AER as compared to TER (Gindre et al., 2016; Lussiana et al., 2017a) could partly explain this greater RFD in AER. But more importantly, several neural aspects, such as the synchronicity of motor unit recruitments and the efficiency of the neural drive, could also significantly impact RFD performance (Maffiuletti et al., 2016). In the present study, the difference observed in EMG activities recorded during RFD between AER and TER is the first clue that such plantar flexors' discrepancy also has a nervous origin. This is not surprising since a strong link is often established between an increase in RFD after training and an increase in the associated EMG activity of the considered muscle group (DelBalso and Cafarelli, 2007). Interestingly, a positive correlation between H-reflex and RFD increase has also been established after plantar flexors training (Holtermann et al., 2007), raising the link between those neural factors and such performances. It was argued previously that the spinal efficiency was a primary factor in enhancing the discharge rate of the motor units needed to improve RFD performance (VanCutsem et al., 1998). In addition, early works of Capaday and Stein (Capaday and Stein, 1987) demonstrated that H-reflexes of the soleus muscles were lower during running than during walking, independently of the level of motor units activity. In other words, differences in H-reflexes can also occur between two different locomotor activities at a given similar EMG activity. Other central mechanisms such as presynaptic inhibition, closely related to muscle lengthening (Duchateau and Enoka, 2008), may be involved. This could also partly explain that the long-term use of different running patterns of AER and TER, and especially the fact that AER runners land in a more plantar flexed position than TER, would lead to long-term changes in spinal excitability. It is admitted that the type of training has a particular influence on medullar network plasticity (Grosprêtre et al., 2018), including interneuronal 
A. SOL

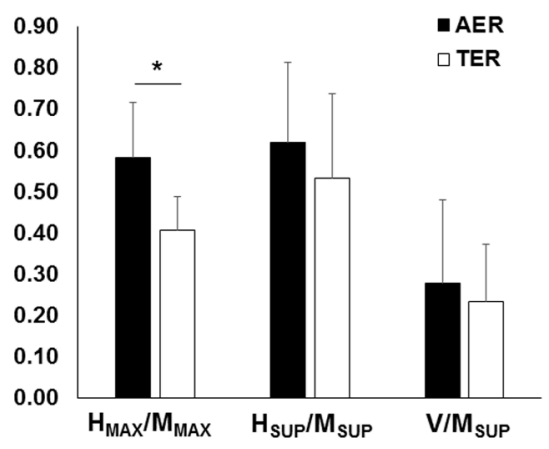

C. $\mathrm{GL}$

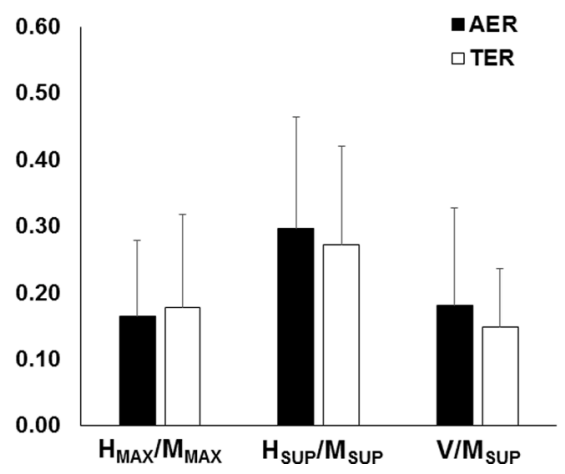

B. $\mathrm{GM}$

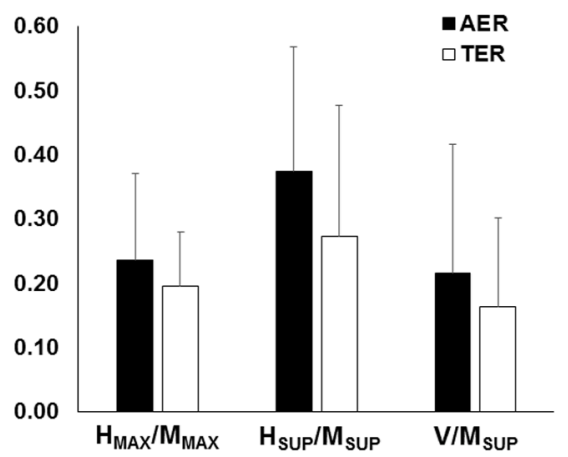

D. REPRESENTATIVE TRACES AER

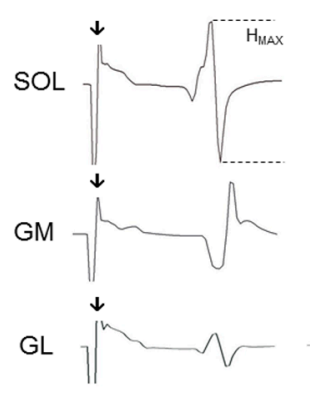

Fig. 3. Electrophysiological ratios of the triceps surae muscles of Aerial (AER) and Terrestrial (TER) runners. Data are depicted as mean \pm SD. Maximal rest H-reflex $\left(\mathrm{H}_{\mathrm{MAX}}\right)$, maximal reflex evoked during maximal contraction $\left(\mathrm{M}_{\mathrm{SUP}}\right)$ and voluntary wave (V) are normalized by their corresponding maximal M-wave $\left(\mathrm{M}_{\mathrm{MAX}}\right.$ or $\left.\mathrm{M}_{\text {SUP }}\right)$. Results are depicted for the three muscles of the triceps surae: soleus (A, SOL), Gatrocnemius Medialis (B, GM) and Gastrocnemius Lateralis (C, GL). D shows the EMG traces of two representative participants. Signals are depicted for maximal H-reflex $\left(\mathrm{H}_{\mathrm{MAX}}\right)$ of SOL, GM and GL. Vertical arrows represent the time of stimulation. *: significant intergroup difference at $\mathrm{P}<0.05$. circuitry that controls and mediates the reflex pathway (Koceja et al., 2004). As a consequence, a greater SOL spinal excitability is usually observed in endurance- as compared to power athletes (Maffiuletti et al., 2001).

At muscle level, no electrophysiological differences have been observed, as evidenced by similar maximal muscle compound potentials (i.e. M-waves) between AER and TER. However, different muscle potentiations have been observed. Such twitch potentiation is attributed to intracellular muscle properties, such as the electrogenic $\mathrm{Na}^{+}-\mathrm{K}^{+}$ pumping efficiency (Cupido et al., 1996) or the intramuscular $\mathrm{Ca}^{2+}$ concentration (Klass, 2004). The fact that TER runners exhibit greater twitch potentiation than AER runners could indicate different intramuscular processes, although the twitch technique is not sufficiently accurate to discriminate one or the other cellular mechanisms.

\subsection{Study limitations}

Such a transversal study does not allow the genetic factor that could have led to recruit participants with particular profiles in the different trained groups to be distinguished. The constitution of groups, in terms of age, level of performance or anthropometric factors, is one of the primary key points of such inter-group comparison. Here, particular care was taken to keep both groups as homogenous as possible, particularly in terms of running experience. The lack of significant inter-group differences in participants' characteristics, limited the drawback of group constitutions. However, it should be noted that the different repartition of males and females between AER and TER could possibly interfere with the results. Some neuromuscular parameters were found to be higher in women than men, such as $\mathrm{H}_{\mathrm{MAX}} / \mathrm{M}_{\mathrm{MAX}}$ ratio (Hoffman et al., 2018). However, the fact that more women were included in TER but greater $\mathrm{H}_{\text {MAX }} / \mathrm{M}_{\text {MAX }}$ was observed in AER, highlights that gender difference did not affect the observed neuromuscular differences, or at worst should have minimized the differences. Overall, due to the lack of a significant global effect of sex on the several tested variables, we suppose that the gender difference had a limited impact on the present results. It should be emphasized that most of the literature investigating sex differences in the previously mentioned performances and neuromuscular characteristics recruited untrained individuals.

Another consequence of such transversal study is the inability to decipher which parameter influences the other. In other terms, the question of whether neuromuscular characteristics determines the running profile or whether using a certain running profile during a long training period shapes the neuromuscular properties as part of a process of adaptation remains open. Previous studies using inter-group comparisons of several types of athletes involved also a control group of untrained participants, with similar characteristics (age, weight, etc) as a "baseline level" regarding neuromuscular parameters (Grosprêtre et al., 2018; Maffiuletti et al., 2001; Tillin et al., 2010). These previous studies tended to show that long term practice of one modality led to significant differences with the control group, in one direction or another. For instance, while triceps surae H-reflex is shown to be reduced in power athletes as compared to control, it is enhanced for endurance athletes (Maffiuletti et al., 2001). Finally, the best to understand the possible links between running profiles and neuromuscular parameters would be a longitudinal approach. Performing a long-term analysis of the neuromuscular parameters evolution with one or the other modality of running could help answering this key question.

\section{Conclusion}

Here, the differences observed between AER and TER runners raised a close link between running forms and neuromuscular and mechanical parameters. AER exhibited higher RFD accompanied by greater SOL EMG activity and H-reflex than TER. The mechanisms underlying different neuromuscular and mechanical profiles of AER and TER depicted a bottom-to-top gradient, significant effects being observed at muscle and spinal levels while no effect were found for any of the supraspinal indexes investigated. These differences seemed to be 
muscle-specific, SOL being the most important muscle to differentiate AER and TER. Therefore, our results extended previous studies showing neuromuscular and mechanical properties differences in different sports by highlighting that neuromuscular and mechanical variability also exists within the same sport, in runners with the same level of expertise and performance that spontaneously chose different running forms. This could be of great importance for training and prophylactic purpose and open new areas of research.

\section{Declaration of Competing Interest}

The authors declare that they have no known competing financial interests or personal relationships that could have appeared to influence the work reported in this paper.

\section{Acknowledgements}

The authors would like to thank the subjects for their time and enthusiasm. We also thank Damien Young for editorial assistance. We confirm that we have read the Journal's position on issues involved in ethical publication and affirm that this report is consistent with those guidelines. None of the authors has any conflict of interest to disclose.

\section{References}

Ahtiainen, J.P., Häkkinen, K., 2009. Strength Athletes Are Capable to Produce Greater Muscle Activation and Neural Fatigue During High-Intensity Resistance Exercise Than Nonathletes. J. Strength Cond. Res. 23, 1129-1134. https://doi.org/10.1519/ JSC.0b013e3181aa1b72.

Arendse, R.E., Noakes, T.D., Azevedo, L.B., Romanov, N., Schwellnus, M.P., Fletcher, G., 2004. Reduced eccentric loading of the knee with the pose running method. Med. Sci. Sports Exerc. 36, 272-277. https://doi.org/10.1249/01. MSS.0000113684.61351.B0.

Bach, T.M., Chapman, A.E., Calvert, T.W., 1983. Mechanical resonance of the human body during voluntary oscillations about the ankle joint. J. Biomech. 16, 85-90. https://doi.org/10.1016/0021-9290(83)90049-0.

Buckthorpe, M., Roi, G.S., 2018. The time has come to incorporate a greater focus on rate of force development training in the sports injury rehabilitation process. Muscles. Ligaments Tendons J. 7, 435-441. https://doi.org/10.11138/mltj/2017.7.3.435.

Capaday, C., Stein, R.B., 1987. Difference in the amplitude of the human soleus $\mathrm{H}$ reflex during walking and running. J. Physiol. 392, 513-522. https://doi.org/10.1113/ jphysiol.1987.sp016794.

Chino, K., Oda, T., Kurihara, T., Nagayoshi, T., Yoshikawa, K., Kanehisa, H. Fukunaga, T., Fukashiro, S., Kawakami, Y., 2008. In vivo fascicle behavior of synergistic muscles in concentric and eccentric plantar flexions in humans. J. Electromyogr. Kinesiol. 18, 79-88. https://doi.org/10.1016/j. jelekin.2006.08.009.

Cupido, C.M., Galea, V., McComas, A.J., 1996. Potentiation and depression of the M wave in human biceps brachii. J. Physiol. 541-550.

DelBalso, C., Cafarelli, E., 2007. Adaptations in the activation of human skeletal muscle induced by short-term isometric resistance training. J. Appl. Physiol. 103, 402-411. https://doi.org/10.1152/japplphysiol.00477.2006.

Driss, T., Lambertz, D., Rouis, M., Vandewalle, H., 2012. Influence of musculo-tendinous stiffness of the plantar ankle flexor muscles upon maximal power output on a cycle ergometre. Eur. J. Appl. Physiol. 112, 3721-3728. https://doi.org/10.1007/s00421 012-2353-5.

Duchateau, J., Enoka, R.M., 2008. Neural control of shortening and lengthening contractions: influence of task constraints. J. Physiol. 586, 5853-5864. https://doi. org/10.1113/jphysiol.2008.160747.

Duclay, J., Martin, A., Robbe, A., Pousson, M., 2008. Spinal reflex plasticity during maximal dynamic contractions after eccentric training. Med. Sci. Sports Exerc. 40, 722-734. https://doi.org/10.1249/MSS.0b013e31816184dc.

Folland, J.P., Buckthorpe, M.W., Hannah, R., 2014. Human capacity for explosive force production: Neural and contractile determinants. Scand. J. Med. Sci. Sports 24, 894-906. https://doi.org/10.1111/sms.12131.

Gindre, C., Lussiana, T., Hebert-Losier, K., Mourot, L., 2016. Aerial and Terrestrial Patterns: A Novel Approach to Analyzing Human Running. Int. J. Sports Med. 37, 25-29. https://doi.org/10.1055/s-0035-1555931.

Grosprêtre, S., Gimenez, P., Martin, A., 2018. Neuromuscular and electromechanical properties of ultra-power athletes: the traceurs. Eur. J. Appl. Physiol. 118, 1361-1371. https://doi.org/10.1007/s00421-018-3868-1.

Grospretre, S., Martin, A., 2014. Conditioning effect of transcranial magnetic stimulation evoking motor-evoked potential on V-wave response. Physiol. Rep. 2 https://doi. org/10.14814/phy2.12191.

Hermens, H.J., Freriks, B., Disselhorst-Klug, C., Rau, G., 2000. Development of recommendations for SEMG sensors and sensor placement procedures. J. Electromyogr. Kinesiol. 10, 361-374.
Hoerzer, S., von Tscharner, V., Jacob, C., Nigg, B.M., 2015. Defining functional groups based on running kinematics using Self-Organizing Maps and Support Vector Machines. J. Biomech. 48, 2072-2079. https://doi.org/10.1016/j. jbiomech.2015.03.017.

Hoffman, M., Norcross, M., Johnson, S., 2018. The Hoffmann reflex is different in men and women. NeuroReport 29, 314-316. https://doi.org/10.1097/ WNR.0000000000000961.

Holtermann, A., Roeleveld, K., Engstrøm, M., Sand, T., 2007. Enhanced H-reflex with resistance training is related to increased rate of force development. Eur. J. Appl. Physiol. 101, 301-312. https://doi.org/10.1007/s00421-007-0503-y.

Johnson, M.A., Polgar, J., Weightman, D., Appleton, D., 1973. Data on the distribution of fibre types in thirty-six human muscles. An autopsy study. J. Neurol. Sci. 18, $111-129$.

Klass, M., 2004. Limiting mechanisms of force production after repetitive dynamic contractions in human triceps surae. J. Appl. Physiol. 96, 1516-1521. https://doi. org/10.1152/japplphysiol.01049.2003.

Koceja, D.M., Davison, E., Robertson, C.T., 2004. Neuromuscular Characteristics of Endurance- and Power-Trained Athletes. Res. Q. Exerc. Sport 75, 23-30. https://doi. org/10.1080/02701367.2004.10609130.

Kyröläinen, H., Komi, P.V., 1994. Neuromuscular performance of lower limbs during voluntary and reflex activity in power- and endurance-trained athletes. Eur. J. Appl. Physiol. Occup. Physiol. 69, 233-239.

Lussiana, T., Gindre, C., Hébert-Losier, K., Sagawa, Y., Gimenez, P., Mourot, L., 2017a. Similar running economy with different running patterns along the aerial-terrestrial continuum. Int. J. Sports Physiol. Perform. 12, 481-489. https://doi.org/10.1123/ ijspp.2016-0107.

Lussiana, T., Gindre, C., Mourot, L., Hébert-Losier, K., 2017b. Do subjective assessments of running patterns reflect objective parameters? Eur. J. Sport Sci. 17, 847-857. https://doi.org/10.1080/17461391.2017.1325072.

Lussiana, T., Patoz, A., Gindre, C., Mourot, L., Hébert-Losier, K., 2019. The implications of time on the ground on running economy: less is not always better. J. Exp. Biol. 222, jeb192047. https://doi.org/10.1242/jeb.192047.

Maffiuletti, Martin, A., Babault, N., Pensini, M., Lucas, B., Schieppati, M., 2001. Electrical and mechanical $\mathrm{H}(\max )$-to- $\mathrm{M}(\max )$ ratio in power- and endurance-trained athletes. J. Appl. Physiol. 90, 3-9.

Maffiuletti, N.A., Aagaard, P., Blazevich, A.J., Folland, J., Tillin, N., Duchateau, J., 2016. Rate of force development: physiological and methodological considerations. Eur. J. Appl. Physiol. 116, 1091-1116. https://doi.org/10.1007/s00421-016-3346-6.

McMahon, T.A., Valiant, G., Frederick, E.C., 1987. Groucho running. J. Appl. Physiol. 62, 2326-2337. https://doi.org/10.1152/jappl.1987.62.6.2326.

Patoz, A., Gindre, C., Mourot, L., Lussiana, T., 2019. Intra and Inter-rater Reliability of the Volodalen $₫$ Scale to Assess Aerial and Terrestrial Running Forms. J. Athl. Enhanc. 8, 4-9.

Phinyomark, A., Osis, S., Hettinga, B.A., Ferber, R., 2015. Kinematic gait patterns in healthy runners: A hierarchical cluster analysis. J. Biomech. 48, 3897-3904. https:// doi.org/10.1016/j.jbiomech.2015.09.025.

Tillin, N.A., Jimenez-Reyes, P., Pain, M.T.G., Folland, J.P., 2010. Neuromuscular Performance of Explosive Power Athletes versus Untrained Individuals. Med. Sci. Sport. Exerc. 42, 781-790. https://doi.org/10.1249/MSS.0b013e3181be9c7e.

Tucker, K.J., Tuncer, M., Türker, K.S., 2005. A review of the H-reflex and M-wave in the human triceps surae. Hum. Mov. Sci. 24, 667-688. https://doi.org/10.1016/j. humov.2005.09.010.

VanCutsem, M., Duchateau, J., Hainaut, K., 1998. Changes in single motor unit behaviour contribute to the increase in contraction speed after dynamic training in humans. J. Physiol. 295-305.

Sidney Grosprêtre received his $\mathrm{PhD}$ in Neurophysiology in 2013. He is now associate professor in the Laboratory C3S "Culture, Sport, Health and Society" (EA4660) in Besancon, France. He teaches neurosciences, physiology, and biomechanics. His main topic of research is about neuromuscular plasticity following acute interventions or training. More specifically, he works mainly on electro-stimulation and motor imagery's impact over the neuromuscular system. Part of his research is also dedicated to investigating the effects of plyometric and eccentric training. Since 2010, he is also founder and president of the French national parkour federation (FPK), a sport based on running and overcoming obstacles mainly in an urban landscape.

Dr. Philippe Gimenez is currently an Associate Professor at the University of Franche Comté, Besançon, France. He received his PhD in Exercise Physiology (2009) from the Faculty of Sports Sciences of the University of Saint-Etienne, France. He was then in a postdoctoral position in the University of Montreal, Montreal, Canada before joining the department Sports-Performance of the Laboratory Culture, Sport, Health and Society (C3S) of the University of Franche-Comté. His main topic of research concerns the physiological and biomechanical determinants of sports performance. More particularly, he is interested in energetic metabolism and associated control mechanisms. He currently works on the effects of combined environnemental stressors to optimize training and recovery.

Adrien Thouvenot obtained a M.Sc. in Sport Science from University of BourgogneFranche Comté (in 2019). He is currently a PhD student within the Research and Development department of Volodalen, Chavéria, France, and the University BourgogneFranche Comté, Besançon, France, on an innovative project funded by a grant CIFRE (no. 2019/0586). Her thesis is concerned with the prevention and rehabilitation of running-related injuries. 
Dr. Aurélien Patoz graduated in Chemistry from Ecole Polytechnique Fédérale de Lausanne, Switzerland and obtained his Ph.D. in Computational Chemistry from the same school. He then obtained a M.Sc. in Sport Science from the University of Lausanne. He is now working as a research associate at the University of Lausanne on an innovative project funded by Innosuisse (grant no. 35793.1 IP-LS). His main research interests focus on running biomechanics. More specifically, his goal is to determine spontaneous running patterns using inertial sensors.

Dr. Thibault Lussiana graduated in Sport Science from University of Franche-Comté, France and obtained his Ph.D. in human movement analysis from the same University. He is now working as a scientist in the Volodalen company (Switzerland). His main research interests focus on the understanding of spontaneous running patterns of individuals.
Dr. Laurent Mourot received his Master degree dedicated to Movement and Health from the University of St Etienne (2000) and his PhD in Sports Sciences from University of Franche Comté (2004). He was a research engineer at the University Hospital of Besançon (Clinical Investigation Center in technological Innovation) before becoming Associate Professor (in 2011) in the Department of Sport Sciences at University of Franche Comté (France). His research interest include integrated physiology and the validation of methods and tools for training/health monitoring in healthy people and patients. He is especially interested in the use of physical activity as a therapeutic tool in cardiovascular disease, including running. 